\title{
INFLUENCE OF CUTANEOUS INVOLVEMENT IN DECIDING HOW TO TREAT PSORIATIC ARTHRITIS
}

Simon Eduardo Gusis, M.D.,1 Sandra Fabiana Montoya, M.D., ${ }^{1}$ Andrea Castelli, M.D., ${ }^{1}$ Kerzberg Eduardo Mario, M.D., ${ }^{1}$ Nora Noemi Kogan, M.D. ${ }^{2}$ Rosana Cyntia Veira, M.D. ${ }^{1}$

1. Department of Rheumatology, Acute General Hospital Jose Maria Ramos Mejia, University of Buenos Aires, Argentina;

2. Department of Dermatology, Acute General Hospital Jose Maria Ramos Mejia, University of Buenos Aires, Argentina

\section{Corresponding author}

Simón Eduardo Gusis, M.D.

Ferrari $2066 \mathrm{C}$

Buenos Aires, Argentina, C.P. 1414

Tel: 5411-48553344

Cell: 54911-31080555

Fax: 5411-45848614

Email: egusis@intramed.net

\section{Disclosures}

The authors have no conflict of interest to declare.

Key words: psoriatic arthritis, psoriasis, treatment, treatment decision, managing therapy, severity

\section{ABSTRACT}

Background: Cutaneous psoriasis precedes joint disease in $84 \%$ of patients with psoriatic arthritis. We believe that skin involvement should be considered when deciding how to treat psoriatic arthritis.

Objective: The goal of our study was to investigate how the severity of skin disease influenced the management of psoriatic arthritis.

Methods: Fifty patients with psoriatic arthritis and cutaneous involvement were studied. The severity of joint and skin involvement was evaluated. Treatment decisions for each patient were based on the most severe presentation between skin and joint involvement.

Results: Treatment decisions were guided and were compared by the most severe skin involvement in $22 / 50$ patients (44\%), by the most severe joint involvement in 16 patients $(32 \%)$, and by both skin and joint involvement in 12 patients $(24 \%)$. There were no statistically significant differences $(p<0.472)$.

Conclusion: Treatment decisions in patients with psoriatic arthritis should be made in conjunction with decisions about skin treatment.

\section{INTRODUCTION}

Psoriasis is a chronic inflammatory skin disease affecting approximately $2 \%$ of the population. ${ }^{1}$ Psoriatic arthritis affects $6 \%$ to $42 \%$ of patients with psoriasis ${ }^{2}$ and is estimated to have a prevalence of $0.1 \%$ to $0.25 \%$ in the United States. ${ }^{3}$ Skin disease precedes joint disease by a mean of 12 years in $84 \%$ of patients with psoriatic arthritis. ${ }^{4}$ 
Treatment strategies are generally indicated separately for psoriatic arthritis and cutaneous involvement in most textbooks, ${ }^{5,6}$ guidelines, ${ }^{2}$ and consensus statements. ${ }^{7}$ Treatment algorithms also are presented separately for each disease. ${ }^{1,8,9}$

The Group for Research and Assessment of Psoriasis and Psoriatic Arthritis (GRAPPA) has recommended that the severity of all domains, including skin disease, be taken into account when deciding how to treat psoriatic arthritis. ${ }^{10}$ In most of our patients, the severity of skin involvement is greater than the severity of joint disease, and this is determinant in deciding how to treat psoriatic arthritis, as many patients would be insufficiently treated if only the severity of joint involvement was considered, having negative consequences on their health. Therefore, we decided to investigate the influence of cutaneous involvement in deciding the treatment of psoriatic arthritis.

\section{OBJECTIVE}

The goal of our study was to investigate how the severity of skin disease influenced the management of psoriatic arthritis.

\section{MATERI AL AND METHODS}

We conducted a cross-sectional observational study of 50 consecutive patients with psoriatic arthritis with concomitant skin disease, evaluated in the outpatient clinics for psoriatic arthritis (Department of Rheumatology) and psoriasis (Department of Dermatology) of the Hospital Ramos Mejia between November 2012 and April 2013. Thirty-five patients were recruited from dermatology and 15 from rheumatology clinics. Both subspecialists reached the final joint treatment decision.

All the patients met the clinical criteria for psoriasis. ${ }^{11}$ The diagnosis of psoriatic arthritis was based on the Classification Criteria for Psoriatic Arthritis. ${ }^{12}$ Four domains of arthritis were analyzed: peripheral arthritis, axial arthritis, enthesitis, and dactylitis. ${ }^{10}$ The following variables were considered in each patient: gender, age, time of progression (in years) of skin and joint disease, the Psoriasis Area Severity Index (PASI), and the severity ofcutaneous ${ }^{13}$ and joint involvement ${ }^{10}$ graded as mild $(\mathrm{M})$, moderate $(\mathrm{Mo})$, or severe $(\mathrm{S})$.

Mild psoriasis was defined as PASI $0<7$, Body Surface Area (BSA) $0<3$, and Dermatology Life Quality Index (DLQI) $0<5$. Moderate psoriasis was defined as PASI $\geq 7<10$, BSA $\geq 3<$ 10 , and DLQI $\geq 5<10$. Severe psoriasis was defined as PASI $\geq 10, B S A \geq 10$, and DLQI $\geq 10.13$

The diagnosis of peripheral arthritis was based on the commitment of the joints of the shoulders, elbows, wrists, hands, hips, knees, ankles, and feet when presented with pain and inflammation of the synovial membrane (synovitis). The diagnosis of axial disease has been based on the presence of two or three of the following criteria: a) inflammatory

back pain, b) limitation of motion of cervical, thoracic, or lumbar spine, c) radiological criteria. Disease activity in the spine can be measured reliably by the Bath Ankylosing Spondylitis Disease Activity Index.

The diagnosis of enthesitis was based on clinical examination, including pain, tenderness, swelling at tendon, ligament, or capsule insertion site by palpation and pressure or by ultrasound with Doppler and magnetic resonance imaging. Dactylitis was defined as uniform swelling of a digit. It is due to synovitis, tenosynovitis, and enthesitis, together with soft tissue edema. ${ }^{10}$ 
In addition, the degree of severity of each domain was classified as mild, moderate, or severe, based on the classification proposed by Ritchlin et al. ${ }^{10}$

Patients who had radiographic damage (erosions and other lesions) were considered to have moderate severity (if radiological damage was mild) or severe (if the radiological damage was moderate). This applies for the four domains of arthritis. ${ }^{10}$

For a patient with different domains of joint involvement, we first evaluated the degree of severity for each domain and then considered the domain with the most severe category. Different situations arose. For example, one patient had moderate peripheral joint involvement, severe axial involvement, mild enthesitis, and mild dactylitis. We deemed that to be severe. A patient with peripheral and axial arthritis with similar degree of severity was considered as having axial involvement.

Then, the severity of cutaneous and joint involvement of each patient with psoriatic arthritis was considered. Three possible scenarios were identified: 1-joint involvement was more severe than cutaneous involvement; 2-joint involvement was as severe as cutaneous involvement; 3-joint involvement was less severe than cutaneous involvement.

Clinical records were analyzed to evaluate how treatments were decided. Patients' preferences and the most severe category of skin and/or joint involvement were considered to guide the treatment strategy. If the severity of joint and cutaneous involvement was similar, treatment was decided according to the presence of mild, moderate, or severe categories for both diseases.

The following combinations and treatments were possible:

- For mild psoriatic arthritis, nonsteroidal antiinflammatory drugs (NSAIDs) were used.

- Disease-modifying antirheumatic drugs (DMARDs) (methotrexate, lef lunomide, cyclosporine, or sulfasalazine) were added for moderate psoriatic arthritis.

- TNF inhibitors (adalimumab, etanercept, and infliximab) were added in severe cases.

Mild psoriasis was treated with topical therapy. Phototherapy and/or systemic treatment (methotrexate, acitretin, or cyclosporine) wasadded in moderate cases. TNF inhibitors and IL12/23 inhibitors (ustekinumab) were used in severe cases (Table 1).

Treatment decisions were compared statistically, according to the highest degree of severity of cutaneous and/or joint involvement. The Chi Squared Yates test was used.

\section{RESULTS}

Twenty-nine of 50 patients (58\%) with psoriatic arthritis and psoriasis were women. Mean age at the time of the study was 51.6 a \pm 14.5 years. The mean time of disease progression was $17.4 \pm 1.1$ years for cutaneous disease and $9.16 \pm 0.8$ years for joint

disease. Mean PASI was $10.6 \pm 1.8$ (Table 2).

Of the 50 patients with psoriatic arthritis, the severity of cutaneous involvement was mild in $14(28 \%)$, moderate in $18(36 \%)$, and severe in $18(36 \%)$. The severity of joint involvement was mild in $11(22 \%)$, moderate in $30(60 \%)$, and severe in nine (18\%). Thirty-nine patients with psoriatic arthritis had radiological lesions.

Treatment decisions were based on the most severe category of cutaneous involvement in $22 / 50$ patients (44\%): six with moderate psoriasis and mild arthritis, two with severe psoriasis 
and mild joint involvement, and 14 with severe psoriasis and moderate arthritis. Treatment was decided according to the most severe category of joint involvement in 16 patients (32\%): nine with moderate arthritis and mild skin involvement, two with severe arthritis and mild skin involvement, and five with severe joint involvement and moderate psoriasis.

Treatment decisions were based on the presence of both diseases in 12 patients (24\%): three with mild cutaneous and joint involvement, seven with moderate cutaneous and joint involvement, and two with severe cutaneous and joint involvement (Table 3).

There were no statistically significant differences $(p<0.472)$ when the therapeutic decisions were compared according to the highest degree of severity cutaneous and/or joint involvement.

\section{DISCUSSION}

When selecting an initial therapy for a patient with psoriatic arthritis, several questions must be answered:

- Should psoriatic arthritis be treated by a rheumatologist and/or a dermatologist?

-Which algorithm should be followed?

The "Guidelines of Care for the Management of Psoriasis and Psoriatic Arthritis" states that dermatologists can treat patients with psoriatic arthritis if the damage is not significant, or they can refer them to a rheumatologist if they feel uncomfortable or inadequately trained. ${ }^{1,14}$

GRAPPA recommendations for the management of psoriatic arthritis were developed by dermatologists and rheumatologists. ${ }^{10}$

Patients with psoriatic arthritis are not mentioned in the "Guidelines on the treatment of psoriasis vulgaris. Update 2011."8

The European League Against Rheumatism recommendations for the management of psoriatic arthritis with pharmacological therapies say that psoriatic arthritis with major skin involvement should be managed by a dermatologist and does not mention that treatments should be indicated by both dermatologists and rheumatologists. ${ }^{9}$

There are multiple possibilities of patients with psoriatic arthritis with joint and skin involvement. Joint domains may present as peripheral arthritis (with a pattern of monoarticular, oligoarticular, or polyarticular involvement), axial involvement, enthesitis, and dactylitis. These domains are not pure, as enthesitis and dactylitis may be present in peripheral and axial arthritis. In turn, peripheral involvement may be present in patients with axial arthritis. ${ }^{15,16}$

Moll and Wright reported that patients with arthritis mutilans often have sacroiliitis, that asymmetrical oligoarticular arthritis is associated with sausage appearance of the digits, and that peripheral arthritis may be present or absent in axial involvement. ${ }^{17}$

The analysis of the clinical presentation with skin and nail involvement is still more complex. In our daily practice, all patients with psoriasis are evaluated by dermatologists and rheumatologists. This approach is useful to prevent delays in the diagnosis of psoriatic arthritis and improve treatment decisions.

In patients who have psoriatic arthritis, we use the following algorithm:

1. Diagnosis of psoriasis: evaluate clinical presentation and degree of severity. 
2. Diagnosis of psoriatic arthritis: evaluate domain and degree of severity.

3. Treatment decision is guided by the domain of joint involvement with the greatest severity and by the severity of cutaneous involvement.

4. Treatment decision is based on the disease with the greatest degree of severity, provided that the patient has agreed with the strategy.

5. The following situations may present: for mild skin and joint involvement, we indicate topical therapy for skin disease and NSAIDs for joint involvement. When moderate arthritis and skin disease are present, treatment depends on the presence of peripheral or axial arthritis.

DMARDs may be used in peripheral arthritis and biologics in axial involvement, in combination with the therapeutic approach of moderate psoriasis. In patients with peripheral arthritis, DMARDs should be combined with first- or second-line systemic drugs for the treatment of psoriasis.

In these cases, the PASI, the American College of Rheumatology 20 , or the Psoriatic Arthritis Response Criteria of each drug should be evaluated to choose the best one for each patient.

Methotrexate and cyclosporine are indicated as first-line agents for moderate joint involvement (level of evidence B) and skin involvement (level of evidence A). Leflunomide and sulfasalazine are considered second-line agents (level of evidence A for both skin and joint involvement). 10 Methotrexate is the drug most commonly used ${ }^{9}$

Biologics are used for severe joint and cutaneous involvement at the initial dose used for skin manifestations. These recommendations mayimprove the therapeutic outcomes.

Yet, this approach is not applicable to a small percentage of patients in whom joint involvement precedes skin compromise. In these patients, the traditional rheumatologic algorithms should be used.

In our study, skin involvement guided the treatment of psoriatic arthritis in 22/50 patients (44\%). Without this decision, these patients would have been insufficiently treated. In 12 patients $(24 \%)$, we decided to treat both joint involvement and skin involvement using biologics (adalimumab and etanercept) at the initial dose used for skin manifestations or combining different systemic drugs in moderate cases. Treatment was decided due to joint involvement in 16 patients (32\%).

In conclusion, treatment decisions in patients with psoriatic arthritis should be accompanied by skin treatment decisions.

\section{REFERENCES}

1. Menter A, Gottlieb A, Feldman SR, et al. Guidelines of care for the management of psoriasis and psoriatic arthritis: section 1. Overview of psoriasis and guidelines of care for the treatment of psoriasis with biologics. J Am Acad Dermatol. 2008;58: 82650 .

2. Gottlieb A, Korman NJ, Gordon KB, et al. Guidelines of care for the management of psoriasis and psoriatic arthritis: section 2. Psoriatic arthritis: Overview and guidelines of care for treatment with an emphasis on the biologics. J Am Acad Dermatol. 2008;58: 851-64. 
3. Guelfand JM, Gladman DD, Mease PJ, et al. Epidemiology of psoriatic arthritis in the population of the United States. J Am Acad Dermatol. 2005;53:573.

4. Gottlieb AB, Kircik L, Eisen D, et al. Use of etanercept for psoriatic arthritis in the dermatology clinic: the experience diagnosing, understanding care, and treatment with etanercept (EDUCATE) study. J Dermatol Treat. 2006;17:343-52.

5. Candia Zúñiga L, Marquez Hernandez J, Espinoza LR. Artritis psoriásica. In AlarcónSegovia D, Molina JL, eds. Tratado Hispanoamericano de Reumatología. ScheringPlough S. A. 2006(1): 585-595.

6. Maldonado Cocco JA, Citera G. Artropatía psoriática. In Maldonado Cocco JA, Citera G. Reumatología. Ediciones Azurra. 2010:462-477.

7. Consenso Latinoamericano de Psoriasis. Guías de Tratamiento.

SOLAPSO.2009. Available at: http://www.solapso.org. Accessed June 22, 2013.

8. Nast A, Boehncke WH, Mrowietz U, et al. Guidelines on the treatment of psoriasis vulgaris. Update 2011. J Dtsch Dermatol Ges. 2011; 9(Suppl 2):S1-104.

9. Gossec L, Smolen JS, Gaujoux-Viala C, et al. European League Against Rheumatism recommendations for the management of psoriatic arthritis with pharmacological therapies. Ann Rheum Dis. 2012;1271: 4-12.

10. Ritchlin CT, Kavanaugh A, Gladman DD, et al. Treatment recommendations for psoriatic arthritis. Ann Rheum Dis. 2009 Sep; 68(9):1387-94.

11. Langley RG, Ellis CN. Evaluating psoriasis with Psoriasis Area and Severity Index, Psoriasis Global Assessment and Lattice System Physician's Global Assessment. J Am Acad Dermatol. 2004;51:563-9.

12. Taylor W, Gladman DD, Helliwel $P$, et al. Classification criteria for psoriatic arthritis: development of new criteria from a large international study. Arthritis Rheum. 2006; 54:2665-73.

13. Parisier D, Bagel J, Gelfand J, et al. National Psoriasis Foundation. Clinical Consensus on Disease Severity. Arch Dermatol. 2007; 143:239-242.

14. Gottlieb A, Korman N, Gordon K, et al. Guidelines of care for the management of psoriasis and psoriasis arthritis. Section 2: Psoriatic arthritis: Overview and guidelines of care for treatment with emphasis on the biologics. J Am Acad Dermatol. 2008; 58(5): 851-64.

15. Heuft-Dorenbosch L, Spoorenberg A, von Tubergen A, et al.Assessment of enthesitis in ankylosing spondylitis. Ann Rheum Dis.2003; 62:127-132.

16. Rothschild BM, Pingitore C, Eaton M. Dactylitis implications for clinicalpractice. Semin Arthritis Rheum. 1998;28:41-7.

17. Moll JMH, Wright V. Psoriatic Arthritis. Semin Arthritis Rheum.1973;3(1):55-78. 